\title{
Role of Vitamin D in rheumatoid arthritis and its association with neuropathic pain: a systematic review and meta-analysis
}

\section{Rozan Elsayed Abd Elfatah}

M.B.B.CH, Rheumatology and Rehabilitation department, Faculty of Medicine - Fayoum University

\section{Abstract}

\section{- Background:}

Rheumatoid arthritis (RA) is the most common autoimmune disease found in about $1 \%$ of adults worldwide. The cause of RA is unknown but there is a combination of genetic and multiple environmental factors dietary resulting in systemic inflammation. Vitamin D not only plays a major role in the regulation of bone mineral homeostasis but also plays a role in in the regulation of the immune response through a different mechanism, these data support a vitamin D deficiency role in the development and progression of RA and other autoimmune diseases. Long-term vitamin D deficiency may lead to decreased immune response and chronic inflammation, which may cause disabling pain. Individuals with vitamin D deficiency often report increased pain perception, which has been referred to hyperinnervation and hypersensitivity in nerve fibers.

\section{- Methods:}

We searched the PUBMED, EMBASE, and Cochrane databases and performed a metaanalysis examining the vitamin D level and prevalence of vitamin $\mathrm{D}$ deficiency in patients with RA compared to healthy controls and the relation between the vitamin D level and disease activity score 28 (DAS28) in RA patients, also we used (LANSS) to evaluate NP in patients with RA.

- Results

Systematic review of 8 articles found that the serum vitamin D level in the RA group was significantly lower than that in the control group. In addition, vitamin D deficiency was more observed in RA patients with neuropathic pain.

\section{- Conclusion}

This systematic review and meta-analysis suggest that vitamin D plays a key role in the susceptibility and activity of RA. Further studies are necessary to elucidate that vitamin D status directly contributes to the pathogenesis of RA and to study the effect of vitamin D supplement in RA patients with neuropathic pain.

- Key words: Vitamin D, Rheumatoid arthritis, neuropathic pain. 


\section{Introduction}

Rheumatoid arthritis (RA) is a chronic autoimmune inflammatory disease that predominantly affects the synovial joints, causing significant morbidity and shortened life expectancy (1). Although its cause is not fully understood, it has been established that genetic and environmental factors contribute to the pathogenesis of RA (2).

Vitamin D (25-hydroxyvitamin D $[25(\mathrm{OH}) \mathrm{D}])$ alters the expression of genes that affect cellular functions such as proliferation, differentiation, apoptosis, and angiogenesis (3). It is known that 1,25-dihydroxy vitamin D3 [1,25(OH)2 D3] inhibits IFN- $\gamma$ secretion and negatively regulates IL-12 production by downregulating NF-kB (4). When administered in vivo,1,25(OH)2D 3 was found to have a preventative effect on autoimmune diseases (5), and other studies have revealed that vitamin D deficiency is linked to many autoimmune diseases $(6,7)$.

The action of vitamin $\mathrm{D}$ is dependent on vitamin $\mathrm{D}$ receptor (VDR), and activation of VDR results in inhibition of pro-inflammatory $\mathrm{T}$ cells and DC differentiation. Furthermore, VDR agonists induce $\mathrm{T}$ regulator and natural killer cells and thus suppress autoimmunity (8), and the
VDR polymorphism has been known to confer susceptibility to RA (9).

A low vitamin D level may increase the RA risk (10). However, studies on vitamin D level in RA patients compared to healthy controls and on the relationship between serum vitamin D levels and RA activity have shown mixed results (11). The reasons for this disparity

may be small sample sizes, low statistical power, and/or clinical heterogeneity (12).

Therefore, in order to overcome the limitations of individual studies, we turned to metaanalysis. The present study aimed to determine serum vitamin $\mathrm{D}$ concentrations and prevalence

of vitamin D deficiency in RA patients compared to healthy controls as well as evaluate its correlation with disease activity and presence of neuropathic pain using the meta-analysis approach.

\section{Methods:}

\section{- Literature search}

We performed a literature search for studies that examined vitamin $\mathrm{D}$ status in RA patients and controls and the relationship between serum vitamin D levels and RA activity. The PUBMED, EMBASE, and Cochrane databases were searched to identify all available articles (October 2021). The 
following key words and subject terms were used in the search: "vitamin D," "level," "deficiency", "rheumatoid arthritis," "RA" and "neuropathic pain" All references cited were also reviewed to identify additional studies not indexed by the electronic databases.

\section{Criteria for meta-analysis}

Studies were considered eligible if: (1) they were case-control studies with patients with RA diagnosed on the basis of diagnosed according to ACR 1987 or ACR/EULAR classification criteria; (2) they provided data on vitamin $\mathrm{D}$ levels and/or vitamin $\mathrm{D}$ deficiency in case and control groups; or (3) NP symptoms such as burning, tingling, numbness and throbbing sensations in any extremity (4) they provided data on the relation between serum vitamin $\mathrm{D}$ levels and RA activity based on disease activity score 28 (DAS28). Disease activity is classified based on the DAS28 value as remission $(<2.6)$, low ( 2.6 to $<3.2)$, moderate $(\geq 3.2$ to 5.1$)$, and high $(\geq 5.1)$. Course of disease, whether or not being new patients, immunosuppression, hormone or calcium treatment maybe have effect on the relationship between vitamin D level and RA, and may cause heterogeneity to affect the authenticity of testing results. However, for inclusion criteria, we did not limit studies based on this information in order to include as many studies as we can to examine a generalized finding.

We excluded studies if: (1) they contained overlapping or insufficient data; or (2) they were reviews or case reports. (3) systemic disease associated with neuropathy (diabetes, hypothyroidism, amyloidosis, liver disease, chronic kidney failure); neurological disease; (5) metabolic bone disease such as hyperthyroidism, hyperparathyroidism or osteomalacia; and (6) use of any medication that could affect vitamin D levels or NP (psychiatric medications such as antidepressants, anxiolytics or sleeping pills).

\section{- Results}

Studies included in the meta-analysis

We identified 269 studies using electronic and manual searching methods, and 33 of these were selected for full text review based on the title and abstract. Nineteen of these were excluded, because they had data on other diseases, no control group, or no vitamin D data. Thus, 14 articles met the inclusion criteria (11).

Thus, A total of 8 articles; including 434 psoriatic cases and 214 controls, were chosen from 33 results in searching the role of the vitamin $\mathrm{D}$ level in the RA and if its deficiency associated with neuropathic pain (NP)?

The first article which examine the development of NP in patients with RA and the relationship between NP and vitamin $\mathrm{D}$ found that vitamin $\mathrm{D}$ deficiency was associated with increased NP in patients with RA where Seventy-five of the eligible patients were female (80.6\%), and 31 $(33.3 \%)$ were diagnosed with NP according to the LANSS.

There was a negative correlation between vitamin $\mathrm{D}$ levels and the LANSS score $(\mathrm{P}=0.001)$. The prevalence of NP was 5.8 times higher among patients with serum 
vitamin $\mathrm{D}$ levels below $20 \mathrm{ng} / \mathrm{mL}$ than in patients with vitamin D levels $\geq 30 \mathrm{ng} / \mathrm{mL}$. Based on the area under curve (AUC) values, we found that serum levels of vitamin $\mathrm{D}$ were a good predictor of NP diagnoses in patients with RA $(A U C=0.71)(\mathbf{1 3})$.

The second one examined the prevalence of vitamin D insufficiency and the associations of vitamin D concentration with disease status in African Americans with rheumatoid arthritis (RA) and showed that Vitamin D insufficiency is common in African Americans with recent-onset RA. Unadjusted associations of circulating vitamin D with baseline pain, swollen joints, and

DAS-28 were explained by differences in season, age, and gender and were not significant in multivariate analyses. In contrast to reports of Northern Europeans with early inflammatory arthritis, there are not strong associations of $25(\mathrm{OH})$ $\mathrm{D}$ concentration with symptoms or disease severity in African Americans with RA (14).

The $3^{\text {rd }}$ article was the first multicenter European survey which add new evidences that vitamin D insufficiency/deficiency is frequent in RA patients with statistically significant differences among several countries as mean serum concentration of $25(\mathrm{OH}) \mathrm{D}$ in RA patients $(17.62 \pm 9.76 \mathrm{ng} / \mathrm{ml})$ was found significantly lower if compared to the levels obtained in matched controls $(18.95 \pm 9.45 \mathrm{ng} / \mathrm{ml})(\mathrm{p}=0.01)$, with statistically significant differences among several European countries. Negative correlations were found between $25(\mathrm{OH}) \mathrm{D}$ serum levels and DAS28-CRP (p b 0.001), RAID ( $\mathrm{p}=0.05)$ and HAQ $(\mathrm{p}=0.04)$ scores in the RA patients' group. Negative correlations were also found in the cohort of enrolled RA patients between $25(\mathrm{OH}) \mathrm{D}$ serum concentrations and SRS $(\mathrm{p}=0.04)$, HRS ( $p=0.02)$ and GRS $(p=0.02)$ domains of the D-PRO questionnaire (15).

The $4^{\text {th }}$ article aimed to assess vitamin D levels in rheumatoid arthritis (RA) patients and to find their relation to clinical parameters, fibromyalgia syndrome (FMS), quality of life (QoL) and disease activity. This study included 63 RA patients and 62 controls. Clinical examination and laboratory investigations were performed. For patients, the Disease Activity Score (DAS-28), QoL index, Health Assessment Questionnaire II (HAQ II) and Modified Larsen score were calculated. 25-OH-vitamin D was measured in patients and controls. This study suggested that special attention is required regarding vitamin D levels in RA patients with FMS and decreased QoL. Vitamin D should be corrected and supplementation considered among the RA management armamentarium (16).

The $5^{\text {th }}$ article evaluated the prevalence of peripheral neuropathy in patients with newly diagnosed rheumatoid arthritis and look for the frequency and pattern of neuropathy in Indian patients with rheumatoid arthritis (RA). Subjects included 
66 patients (M 13: F 53) with mean age of 42 $( \pm 13.42)$ years and median disease duration of 36 months (IQR-13.5, 60). Sensory symptoms were present in 9 patients (13.6\%). None had motor symptoms. On neurological examination, 16 patients had sensory $(24.2 \%)$ and 6 (9.09\%) had motor abnormalities. Nerve conduction studies showed abnormality in 25 patients (37.87\%). Evidence of entrapment neuropathy was found in 6 patients $(9.09 \%$; 5 patients with median nerve involvement [unilateral, 3 and bilateral, 2] and 1 patient with unilateral ulnar nerve involvement), 3 patients had only sensory neuropathy, 5 had mixed sensory motor and 3 had only motor neuropathy. Eight patients (12.12\%) had only small fiber neuropathy as detected by sympathetic skin response and quantitative sensory testing. This study shows high prevalence of subclinical neuropathy in Indian patients with RA. This may be an important contributor to disability (17).

The $6^{\text {th }}$ article was performed to evaluate vitamin $\mathrm{D}$ status in patients with RA and to assess the relationship between vitamin D levels and disease activity. In the cohort of 44 patients with RA $25(\mathrm{OH}) \mathrm{D} 3$ levels were found to be low compared with the control group, 25(OH)D3 being $15.26 \pm$
$1.07 \mathrm{ng} / \mathrm{ml}$ [mean \pm standard error of the mean (SEM)] and $25.8 \pm 1.6 \mathrm{ng} / \mathrm{ml}$ in the patient and control group respectively (Student's $\mathrm{t}$ test, $\mathrm{p}<0.001$ ). CRP $7.6 \pm 1.57$ $\mathrm{mg} /$ liter (mean $\pm \mathrm{SEM}$ ) (normal values $<3$ $\mathrm{mg} / \mathrm{litre}$ ) and ESR was $38.0 \pm 4.6 \mathrm{~mm} / \mathrm{h}$ (mean \pm SEM) in the group of patients with RA. Levels of $25(\mathrm{OH}) \mathrm{D} 3$ were found to be negatively correlated to the DAS28, the correlation coefficient being -0.084 . Levels of $25(\mathrm{OH}) \mathrm{D} 3$ were also found to be negatively correlated to CRP and ESR, the correlation coefficient being -0.115 and -0.18 , respectively (18).

The $7^{\text {th }}$ article was conducted to determine if there is a link between vitamin D deficiency and chronic pain, a systematic review of the evidence would be expected to uncover two things: firstly, an inverse association between pain and $25-\mathrm{OH}$ vitamin D levels and, secondly, a demonstrable benefit of vitamin $\mathrm{D}$ treatment. They identified 22 relevant studies that reported mean 25-OH vitamin D levels and/or investigated the results of vitamin $D$ treatment in patients with chronic pain conditions. Five were randomized double blind trials of vitamin D treatment. Eight studies with weaker designs more prone to bias also evaluated vitamin D treatment; two 
were randomized but not double-blind and six were case series. Nine purely observational studies were without treatment. These 23 data sets ranged in size from 5 to 3459 patients. The total number of patients in "pain" and "control" groups was 8644; 58\% were women. Few studies actually measured vitamin D status, and there was no common definition of what constituted deficiency. Finally, it recommended the need to better understand how Vitamin D could generate or maintain pain, especially musculoskeletal pain (other than osteomalacia, which is known to be due to vitamin D deficiency). This should lead to better and more focused research (19).

The last article included in my study was performed to determine if there is an association between mean 25(OH)D concentration (primary aim), or proportion of hypovitaminosis D (secondary aim), and pain conditions in observational studies. Eighty-one observational studies with a total of 50834 participants were identified. Compared with controls, mean $25(\mathrm{OH}) \mathrm{D}$ concentration was significantly lower in patients with arthritis (mean difference (MD): $-12.34 \mathrm{nmol} / \mathrm{l} ; \mathrm{P}<$ 0.001), muscle pain (MD: $-8.97 \mathrm{nmol} / \mathrm{l} ; \mathrm{P}=$ 0.003 ) and chronic widespread pain (MD: $-7.77 \mathrm{nmol} / \mathrm{l} ; \mathrm{P}<0.001)$, but not in patients with headache or migraine (MD: $-2 \cdot 53 \mathrm{nmol} / \mathrm{l}$; $\mathrm{P}=0 \cdot 06$ ). The odds of vitamin $\mathrm{D}$ deficiency were increased for arthritis, muscle pain and chronic widespread pain, but not for headache or migraine, compared with controls. A significantly lower $25(\mathrm{OH}) \mathrm{D}$ concentration was observed in patients with arthritis, muscle pain and chronic widespread pain, compared with those without. These results suggest that low 25(OH)D concentrations may be associated with pain conditions (20).

\section{Discussion}

A total of 8 articles were chosen from 33 results in searching role of vitamin $D$ in rheumatoid arthritis and its association with neuropathic pain. All of studies found that RA patients had lower vitamin D level than control subjects, in addition, some studies found that vitamin D deficiency was more observed in RA patients with neuropathic pain.

\section{Meta-analysis of this study revealed}

\section{that:}

- RA patients had lower vitamin D level than control subjects as regard the mean value of vitamin D blood level.

- The prevalence of NP was 5.8 times higher among patients with serum vitamin D levels below

$20 \mathrm{ng} / \mathrm{mL}$ than in patients with vitamin $\mathrm{D}$ levels $\geq 30 \mathrm{ng} / \mathrm{mL}$.

- Vitamin D is considered as a clinical biomarker for RA. 
- Vitamin D deficiency may be linked to disease severity in RA.

- Low vitamin D concentration is associated with arthritis, muscle pain and chronic widespread pain.

- Vitamin D supplementation may be needed both for the prevention of osteoporosis as well

as for pain relief in patients with RA.

\section{Conclusion}

In conclusion, this review summarizes the literature on the effect of different vitamin D levels and it was concluded that its deficiency could influence the physical health and quality of life, moreover it may be one of the causes leading to worsening of RA clinically and also affected the laboratory investigations. In addition, vitamin D deficiency was more observed in RA patients with neuropathic pain as vitamin D is considered an essential neuro-steroid with a major role in central and peripheral nervous system physiology, and its deficiency contribute to hyperinnervation and hypersensitivity in nerve fibers.

\section{References:}

\section{Harris ED JR. (1990): Rheumatoid} arthritis. Pathophysiology and implications for therapy. N Engl J Med; 322: 1277-1289.

2. Choi SJ, Rho YH, Ji JD, Song GG, Lee YH (2006): Genome scan meta-analysis of rheumatoid arthritis. Rheumatology (Oxford); 45:166-170.

3. Cantorna MT (2000): Vitamin D and autoimmunity: is vitamin $\mathrm{D}$ status an environmental factor affecting autoimmune disease prevalence? Proc Soc Exp Biol Med; 223: 230-233.

4. Boonstra A, Barrat FJ, Crain C, Heath VI, Savelkoul HF, O'garra A (2001): 1alpha,25- Dihydroxyvitamin $\mathrm{d} 3$ has a direct effect on naive CD4(+) $\mathrm{T}$ cells to enhance the development of Th2 cells. I Immunol; 167: 4974-4980.

5. Koizumi T, Nakao Y, Matsui $T$ et al. (1985): Effects of corticosteroid and 1,24Rdihydroxy-vita-min D3 administration on lymphoproliferation and autoimmune disease in MRL/MP-lpr/lpr mice. Int Arch Allergy Appl Immunol; 77: 396-404.

6. Kamen D, Aranow C (2008): Vitamin D in systemic lupus erythematosus. Curr Opin Rheumatol; 20: 532-537.

\section{Cutolo M, Otsa K, Paolino S, Yprus M,} Veldi T, Seriolo B (2009): Vitamin D involvement in rheumatoid arthritis and systemic lupus erythaematosus. Ann Rheum Dis;

68: 446-7.

8. Wen H, Baker JF (2011): Vitamin D, immunoregulation, and rheumatoid arthritis. J Clin Rheumatol; 17: 102-107.

9. Lee YH, Bae SC, Choi SJ, Ji JD, Song GG (2011): Associations between vitamin D receptor polymorphisms and susceptibility to rheumatoid arthritis and 
systemic lupus erythematosus: a metaanalysis. Mol Biol Rep; 38: 3643-3651.

\section{Gatenby $P$, Lucas $R$, Swaminathan $A$} (2013): Vitamin D deficiency and risk for rheumatic diseases: an update. Cur Opin Rheumatol; 25: 184-191.

\section{Grazio S, Naglic DB, Anic B Et al.} (2015): Vitamin D serum level, disease activity and functional ability in different rheumatic patients. Am J Med Sci; 349: 46-55.

\section{Brance ML, Brun LR, Lioi S, Sanchez} A, Abdala M, Oliveri B (2015): Vitamin D levels and bone mass in rheumatoid arthritis. Rheumatol Int; 35: 499-505.

\section{Yesil H, Sungur U, Akdeniz S, Gurer} G, Yalcm B. \& Dundar U. (2018): Association between serum vitamin D levels and neuropathic pain in rheumatoid arthritis patients: A cross-sectional study. International journal of rheumatic diseases, 21(2), 431-439.

14. Craig S M, Yu F, Curtis JR, Alarcón GS, Conn DL, Jonas B.... \& Mikuls TR. (2010): Vitamin D status and its associations with disease activity and severity in African Americans with recent-onset rheumatoid arthritis. The Journal of rheumatology, 37(2), 275-281.

15. Vojinovic J, Tincani A, Sulli A, Soldano S, Andreoli L, Dall'Ara F. ... \& Cutolo M. (2017): European multicentre pilot survey to assess vitamin D status in rheumatoid arthritis patients and early development of a new Patient Reported Outcome questionnaire (DPRO). Autoimmunity reviews, 16(5), 548554.

16. Gheita TA, Sayed S, Gheita HA \& Kenawy SA. (2016): Vitamin D status in rheumatoid arthritis patients: relation to clinical manifestations, disease activity, quality of life and fibromyalgia syndrome. International journal of rheumatic diseases, 19(3), 294-299.

17. Aneja R, Singh MB, Shankar S, Dhir V, Grover R, Gupta R \& Kuma A. (2007): Prevalence of peripheral neuropathy in patients with newly diagnosed rheumatoid arthritis. Indian Journal of Rheumatology, 2(2), 47-50.

18. Kostoglou-Athanassiou I, Athanassiou P, Lyraki A, Raftakis I \& Antoniadis C. (2012): Vitamin D and rheumatoid arthritis. Therapeutic advances in endocrinology and metabolism,3(6), 181187.

19. Straube S, Moore AR, Derry $S$ \& McQuay HJ. (2009): Vitamin D and chronic pain. Pain, 141(1), 10-13.

20. Wu Z, Malihi Z, Stewart AW, Lawes CM \& Scragg R. (2018): The association between vitamin D concentration and pain: a systematic review and meta-analysis. Public health nutrition, 21(11), 2022-2037. 